\title{
Maximum Time and Distance needed for Pair Production inside Atom, Analytical and Theoretical
}

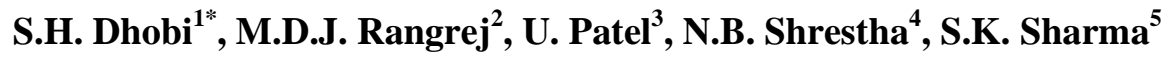 \\ ${ }^{1,3}$ Innovative Ghar Nepal Laboratory, Innovative Ghar Nepal, Lalitupur-44700, Nepal \\ 2, 4,5 Department of Physics, Tribhuvan University, Kathmandu-44600, Nepal \\ *Corresponding Author: saddam@ran.edu.np, Tel.: +977-9808100285
}

Available online at: www.isroset.org

Received: 27/Jan/2020, Accepted: 20/Feb/2020, Online: 28/Feb/2020

\begin{abstract}
In pair production, particle and antiparticle are generated when energy form mass and mass form dipole and then separation take place in stronger nuclear filed. After the formation of mass, it goes on oscillation and oscillation causes the dipole creation on mass which further goes on separation from each other as $Q_{1} E_{X}^{3} \ll Q_{2} E_{(X / X)}^{3}$ and moreover the forces $F_{32}>>F_{31}$. These two component play major role for the separation of the masses in oscillation time having frequency (f). Also with the help of size of nucleus and atom we calculate the maximum distance and maximum time for pair production $9.99 \times 10^{-11} \mathrm{~m}$ and $3.33 \times 10^{-19} \mathrm{~s}$, respectively.
\end{abstract}

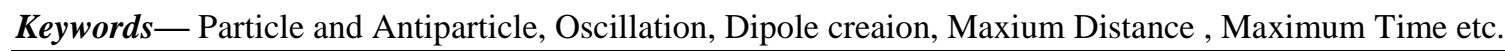

\section{INTRODUCTION}

Considering a particle of mass ' $\mathrm{m}$ ' and charge ' $\mathrm{q}$ ' is accelerated from rest through a voltage $\mathrm{V}$, then energy given to the electron is $\mathrm{qV}$. If all this energy is converted into motion then we can written as, $q V=\frac{1}{2} m v^{2}$, where $\mathrm{v}$ is velocity of mass. The particle motion is governed by the Lorentz force via the following equation, $\frac{d\left(M_{0} P \vec{V}\right)}{d t}=\vec{F}^{e x t}=e(\vec{E}+\vec{v} \times \vec{B})$, where $\mathrm{M}_{0}$ is rest mass, $\gamma$ is relativistic factor, and $\vec{v}$ is particle velocity. This equation help to calculate trajectory of the charge moving through any e.m field [2]. The value of the electric field $\vec{E}$ created by charge separation is, as we have seen, proportional to the Separation length $(\mathrm{x})$ is given as, $E=\frac{E}{\varepsilon_{0}} n x$. Also the force carried or experience by the electron or particle in field $E$ is given as, $=e E=\frac{e^{2}}{\varepsilon_{0}} n x=m_{e} \frac{d^{2} x}{d t^{2}}$. This is the equation of a harmonic oscillator with the Eigen frequency $\omega_{p e}=\left(\frac{n e^{2}}{\varepsilon_{0} m_{\underline{R}}}\right)^{\frac{\pi}{2}}$.This so-called (angular) electron plasma frequency [3].

The minimum energy needed for the pair-production is supplied by photon is $2 \mathrm{mc}^{2}$ ( $\mathrm{m}$ is the mass of the electron/positron). The photon interacts with the electromagnetic field of the nucleus to produce the $\mathrm{e}^{-} \mathrm{e}^{+}$pair. Some reaction for pair production is given as $\gamma \rightarrow e^{+}+e^{-}$and $\gamma \rightarrow e^{+}+e^{-}+\gamma$.
The probability for pair production to occur is higher for photons with more energy and increased with atomic number of the matter as $\mathrm{Z}^{2}$ [4].

\section{REVIEW}

On considering the spherical shape of nucleus, radius of nucleus from liquid drop model is given as $R=R_{0} A^{1 / 3}$ where, $\mathrm{R}_{0} \approx 1.22 \mathrm{fm}$ [5], [6]. The radius $\mathrm{R}$ of the nucleus is approximately proportional to $\mathrm{A}^{1 / 3}$, and the relation that fits the experimental data best for relation, $R=R_{0} A^{1 / 3}$. The constant $\mathrm{R}_{0}$ is equal to $1.5 .10^{-13} \mathrm{~cm}$. However, $\mathrm{R}_{0}$ varies within an interval depending on the element [7].

Solid Spherical shape of the nucleus with a precision given as $R_{n}=(1.25 \pm 0.051) \mathrm{A}^{1 / 3} \mathrm{fm}$, where $\mathrm{A}$ is atomic number. This Equation gives the functional dependence the nucleus radius $R_{n}$ in terms of atomic number [8]. If there is an electric force acting on an object having a charge $\mathrm{q}$, then the electric field at that point is given by $\mathrm{E}=\mathrm{F} / \mathrm{q}$. The charge $\mathrm{Q}$ produces an electric field which in turn produces a force on the charge $\mathrm{q}$. The force on $\mathrm{q}$ is expressed as, $\mathrm{F}=$ $\mathrm{K} \mathrm{qQ} / \mathrm{r} 2=\mathrm{q}(\mathrm{KQ} / \mathrm{r} 2)=\mathrm{q}$ E. The units of $\mathrm{E}$ are Newton's per Coulomb (units = N/C) [9], [10].

\section{MATERIALS AND METHODS}

When high energy photon enter inside an atom and enter in nucleus field, which is vary inside the atom at different point, the nucleus field resist the speed of photon and finally the photon goes on the rest, then from relativity theory we have, 


$$
M=\frac{M_{0}}{\sqrt{1-\frac{v^{2}}{c^{2}}}} \cdots \cdots \cdots \cdots \cdots(1)
$$

Where $\mathrm{M}_{\mathrm{o}}$ is mass of photon at rest, $\mathrm{v}^{2}$ velocity of photon when enter inside an atom, $\mathrm{c}^{2}=$ velocity of light and $\mathrm{M}$ is the mass of photon at any instant $[11,12,13]$. In our case, we are considering the speed $\mathrm{v}=0$ at a certain distance for nucleus in high nuclear field, due to resist of nucleus field. Then from equation (1) we have, $\mathrm{M}=\mathrm{M}_{0}[14]$.

In this ways, we can also introduce the energy of photon at difference situation i.e. $\mathrm{Mc}^{2}$, where $\mathrm{v}$ is relativistic velocity and $\mathrm{M}_{\mathrm{o}} \mathrm{c}^{2}$ is rest energy of photon.

$M c^{2}=\frac{M_{0} c^{2}}{\sqrt{1-\frac{V^{2}}{c^{2}}}} \cdots \cdots \cdots \cdots \cdots(2)$
$E=\frac{E_{0}}{\sqrt{1-\frac{V^{2}}{c^{2}}}} \cdots \cdots \cdots \cdots \cdots \cdots(3)$

After the formation of mass at certain point mass goes on acceleration and moreover this phenomena take place in nucleus field then, we have the can applied a relation

$$
M a=q E_{\mathbb{N}}
$$

Where $\mathrm{a}$ is the acceleration of the mass after the formation and $\mathrm{q}$ charge of mass formed from photon and $\mathrm{E}_{\mathrm{N}}$ is resultant nucleus field of nucleus and is not homogeneous. As, $\mathrm{Ma}=\mathrm{qE}$ for acceleration of mass $\mathrm{m}$ in field $\mathrm{E}$, from (4)

$$
\alpha=\frac{q E_{N}}{M} \ldots \ldots \ldots m \ldots
$$

Where a, is the acceleration of mass after formation in nucleus filed. Also the oscillation as harmonic oscillation of this mass is $a=-(2 \pi f)^{2} z$

Where $\mathrm{f}$ is oscillation frequency and $\mathrm{x}$ is the displacement of mass from it center mass. On before the formation of dipole in mass, we have from (5) and (6)

$$
\begin{aligned}
& \frac{q N_{f}}{M}=-(2 \pi f)^{2} x \\
& -\frac{q N_{f}}{4 \pi M x}=f^{2} \\
& f=-\sqrt{\frac{q N_{f}}{4 \pi M x}}
\end{aligned}
$$

This is the relation for the frequency at which mass $M$ is oscillation in nuclear field.

Since the formation of mass take place at a position or a point in nuclear field. At this point the mass goes on free oscillation because it is a boundary point where repulsion of positive charge nuclear field resist or oppose the motion of mass. Due to this oscillation dipole is creation in oscillation mass. In this oscillation dipole is created in such a way that positive part of mass is away from nucleus and negative part is towards nucleus in nuclear field.

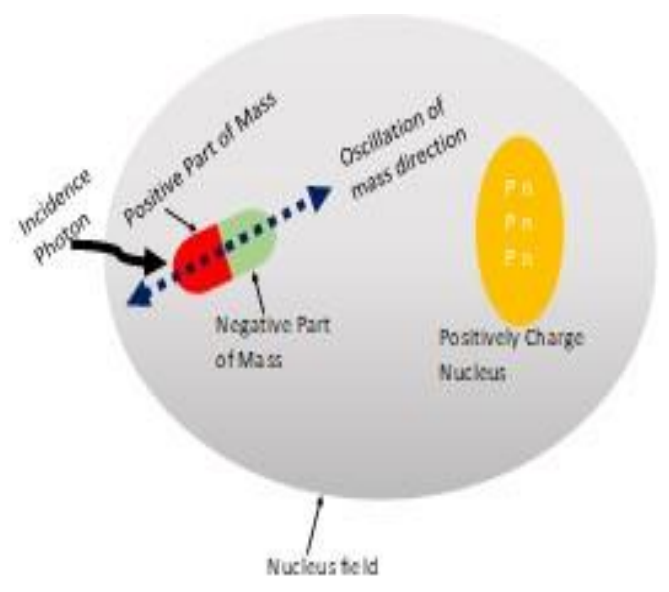

Figure 1: Oscillation of mass in nucleus field or nuclear field at oscillation point or boundary point.

The formation of dipole in a mass oscillation start at the boundary point, here boundary point is a point where the mass are found and depends upon the size of nucleus.

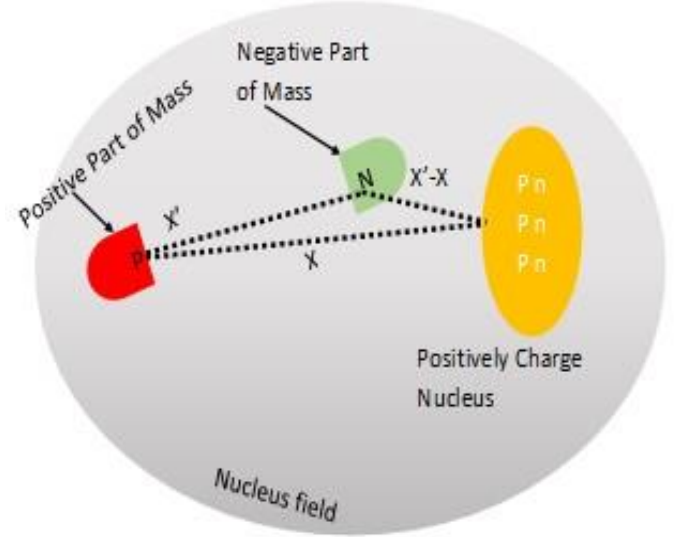

Figure 2: Creation of Dipole and the separation distances from each other.

Here $\mathrm{X}^{\prime}$ is the maximum distance at which $\mathrm{Q}_{1}$ and $\mathrm{Q}_{2}$ are separated to leave the nucleus field. This separation distance causes the delay or difference in time for particle and antiparticle, in pair production.

$\mathrm{X}$ is the distance of positive charge, $\mathrm{Q}_{1}$ and $\mathrm{Q}_{2}$, moreover this distance is the maximum distance from where positive charge $\left(Q_{1}\right)$ are separated from negative charge $\left(Q_{2}\right)$ and then leave the nucleus field.

$\mathrm{X}-\mathrm{X}^{\prime}$ is the distance of positive and negative charge from where $\mathrm{Q}_{2}$ leave nucleus field. This distance is too small or short in comparison of $X^{\prime}$. The negative mass goes repulsion from the nucleus field and leave the nucleus field after the positive mass leave.

$\mathrm{P}$ is center of mass of positive charge and $\mathrm{N}$ is center of mass of negative charge. The electric field produced by the charge $\mathrm{Q}$ at a point $\mathrm{r}$ is given as $E=\frac{K Q}{r^{2}}$ where, $K=\frac{1}{4 \pi \varepsilon_{00}}$, from the figure we have electric field by $\mathrm{Q} 3$ at point $\mathrm{X}$ or at a distance $\mathrm{X}$ as 
$E_{X}^{\mathrm{a}}=\frac{K Q_{a}}{X^{2}}$

Similarly, electric field by $\mathrm{Q} 3$ at point $\left(\mathrm{X}^{\prime}-\mathrm{X}\right)$ or at a distance $\left(\mathrm{X}^{\prime}-\mathrm{X}\right)$

$E_{X-X}^{a}=\frac{K Q_{a}}{\left(X^{y}-X\right)^{2}}$

Or, from (8) and (9) we have,

$$
\begin{aligned}
& \frac{E_{X}^{\mathrm{a}}}{E_{(X / X)}^{\mathrm{a}}}=\frac{K Q_{3}}{X^{2}} \times \frac{\left(X^{t}-X^{t}\right)^{2}}{K Q_{3}} \\
& \frac{E_{X}^{3}}{E_{[X ! X]}^{2}}=\frac{\left(X^{y}-X\right)^{2}}{X^{2}} \\
& \frac{E_{X}^{\mathrm{a}}}{E_{[X-X]}^{a}}=\left(\frac{X^{y}-X}{X}\right)^{2}
\end{aligned}
$$

Since in our consideration we have $\left(X^{-} X^{\prime}\right)<X$ because $(X-$ $\left.\mathrm{X}^{\prime}\right)$ is the distance of negative part and $\mathrm{X}$ distance of positive charge, and the orientation of negative part is towards the nucleus (Positively charge) and positive charge away from nucleus. Therefore

$\left(\frac{X^{v}-X}{X}\right)<1$

Now from (10) and (11). We have,

$\frac{E_{X}^{a}}{E_{[X !-X)}^{a}}=\left(\frac{X^{J}-X}{X}\right)^{2} \ll 1$

$E_{\mathbb{X}}^{\mathrm{a}} \ll E_{(\mathbb{N}-1)}^{\mathrm{a}}$

This show that the $\mathrm{Q}_{2}$ is in high electric field while $\mathrm{Q}_{1}$ is in less electric field this also implies that the force between the $\mathrm{Q}_{2}$ and $\mathrm{Q}_{3}$ is greater than that of $\mathrm{Q}_{1}$ and $\mathrm{Q}_{3}$. This also show the oscillation of mass goes on two electric field having different magnitude and this causes the formation of dipole in formation mass from high energy incidence photon.

Since we have $\mathrm{F}=\mathrm{qE}$ then from equation (12) we have $Q_{1} E_{X}^{2}\left\langle Q_{2} E_{(X-X)}^{2}\right.$

This show that acting between $Q_{1}$ and $Q_{3}$ is less than that of force acting in between $\mathrm{Q}_{2}$ and $\mathrm{Q}_{3}$.

Case I: If $\mathrm{Q}_{1}$ and $\mathrm{Q}_{2}$ are the same charge (negative) and $\mathrm{Q}_{3}$ is positive then Force between $\mathrm{Q}_{1}$ and $\mathrm{Q}_{3}, \mathrm{~F}_{31}$ is greater than force between $Q_{2}$ and $Q_{3}, F_{32}$ i.e. $F_{32}>>F_{31}$, this is because of distance.

Case II: If $\mathrm{Q}_{1}$ positively charge and $\mathrm{Q}_{2}$ negatively charge and $\mathrm{Q}_{3}$ is positive then Force between $\mathrm{Q}_{1}$ and $\mathrm{Q}_{3} \mathrm{~F}_{31}$ is greater than force between $\mathrm{Q}_{2}$ and $\mathrm{Q}_{3}, \mathrm{~F}_{32}$ i.e. $\mathrm{F}_{32}>>\mathrm{F}_{31}$, this because of charge nature (either positive or negative) and distance separation of the charges.

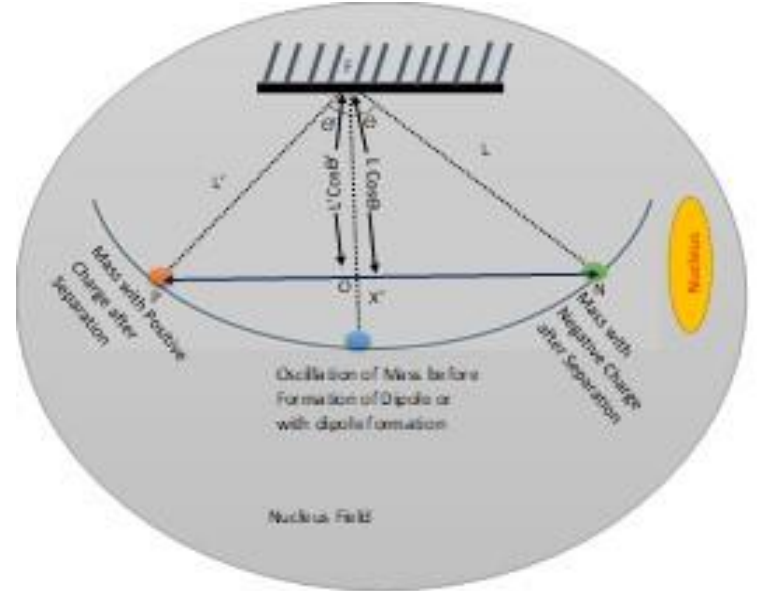

Figure 3: Separation of positive and negative masses during oscillation in nucleus field.

Since $X^{\prime}$ is the distance separation and taking triangle $\mathrm{POF}$ and $\mathrm{ONF}$, we have

$P F^{2}=P O^{2}+O F^{2}$

[From triangle

$\mathrm{POF}]$

$P O^{2}=P F^{2}-O F^{2}$

$$
P O=\sqrt{\left(P F^{2}-O F^{2}\right)}
$$

On putting the value of $\mathrm{PF}=\mathrm{L}$ ' and $\mathrm{OP}=\mathrm{L}$ ' $\cos \Theta^{\prime}$ from figure we get,

$$
\begin{gathered}
P O=\sqrt{\left(L^{\prime 2}-L^{s} \cos ^{2} \theta^{\prime}\right)} \\
P O=L^{y} \sqrt{1-\cos ^{2} \theta^{y}} \\
P O=L^{y} \sin \theta_{x}
\end{gathered}
$$

Similarly we can get,

NO $=\sqrt{\left(N F^{2}-O F^{2}\right)}$

And

$N O=L \sin \theta$

Now from figure 3

$X^{v}=P O+N O=L^{s} \sin \theta^{v}+L \sin \theta$

Therefore,

$X^{s}=L^{s} \sin \theta^{\theta}+L \sin \theta$

The atomic radii of an atoms are from 0.2 Angstrom to 6 Angstrom [15]. The typical atom has a diameter of $10^{-10}$ meters. The typical nucleus has an average diameter of $10^{-}$ 15 meters. This means, that on average, an atom has a diameter that is $10^{5}$ or 100,000 times bigger than its nucleus [16].

In general the radius of an atom is in the range of angstrom and nuclues radius is measeure in femtometer. Since the pair prodction take place inside an atom then the maxium distance of pair production X', which is also generalized as the separation of positive and negrative mass.

We have from pair production, minimum amount energy of photon is about $2 \mathrm{~m}_{\mathrm{e}} \mathrm{c}^{2}=1.02 \mathrm{MeV}$ and velocity is about $3 \mathrm{x}$ $10^{8} \mathrm{~m} / \mathrm{s}$. Now the maxium distance between nuclues and atom surface is taken as (radius of atom-radius of nuclues $)=10^{-10}-10^{-15}=9.99 \times 10^{-11} \mathrm{~m}$ and this is equal to $X^{\prime}$. Therfore $X^{\prime}=9.99 \times 10^{-11} \mathrm{~m}$ is the distance where the pair 
production take place after entering the photon inside the atoms.

Also from $\quad$ equation $\quad$ (15) we have,
$9.99 \times 10^{-11} m=L^{s} \sin \theta^{s}+L \sin \theta \ldots \ldots \ldots \ldots \ldots \ldots$
(16)

Also the maximum speed of photon (c) is $3 \times 10^{8} \mathrm{~m} / \mathrm{s}$.

From this we can find the maximum time for pair production, for this we have

$$
\begin{gathered}
\text { Velocity }(V)=\frac{\text { Distance Travel }(D)}{\text { Time Taken }(T)} \\
\text { Time Taken }=\frac{\text { Distance Travel }}{\text { Velocity }}=\frac{9.99 \times 10^{-11} \mathrm{~m}}{3 \times 10^{8} \mathrm{~m} / \mathrm{sec}}=3.33 \times 10^{-19} \mathrm{sec}
\end{gathered}
$$

Hence the maximum time required for pair production is about $3.33 \times 10^{-19} \mathrm{~s}$.

\section{RESULTS AND DISCUSSION}

On considering the radius of an atom one angstrom and nucleus one femtometer. We Calculated the distance inside an atom in which entering photon goes to created article and antiparticle by subtraction the radius of an atom and nucleus of an atom because pair production don't take place in nucleus. This means all the phenomena of pair production take place in field of nucleus, subtraction need because nucleus consider as solid sphere and the reference point to calculate the radius of both atom ad nucleus is same. In this way we found maximum distance in which pair production phenomena take place is about $9.99 \times 10^{-}$ ${ }^{11} \mathrm{~m}$ which is also a maximum distance for the separation of positive and negative mass created in such phenomena. Since velocity of light is $\mathrm{c}$ and the minimum amount of energy required for pair production phenomean is $2 \mathrm{mc}^{2}$, where $\mathrm{c}$ is light velocity, with the help of this we found the maximum velocity of positive and negative mass $\mathrm{c}$. This $\mathrm{c}$ help to calculate the the time separation of positive and negative mass about $3.33 \times 10^{-19} \mathrm{~s}$. This is the time for sepration of positive and negative mass that is positive mass leave faster by $3.33 \times 10^{-19} \mathrm{~s}$ than that of negarive mass or negative mass leave delay by $3.33 \times 10^{-19} \mathrm{~s}$ than that of positive mass.

On other hand the role of electric field and force play an important role to create the dipole in neutral mass and separation of them i.e. the electric field of nucleus acting on $\mathrm{Q}_{1}$ is less than that of $\mathrm{Q}_{2}$ becauses of distance and charge. Here distance indicate the speration of $\mathrm{Q}_{1}, \mathrm{Q}_{2}$ and $\mathrm{Q}_{3}$ and in our study separation of $\mathrm{Q}_{1}$ and $\mathrm{Q}_{3}$ is greater than $\mathrm{Q}_{2}$ and $\mathrm{Q}_{3}$ also for the charge $\mathrm{Q}_{1}$ and $\mathrm{Q}_{3}$ positively charge while $\mathrm{Q}_{2}$ and $\mathrm{Q}_{3}$ are negativly charge.

\section{CONCLUSION}

Therfore we conclued, that the maxium distance for pair praoduction phaenomeana is about $9.99 \times 10^{-11} \mathrm{~m}$ and time to leave positive mass inside the atom is $3.33 \times 10^{-19} \mathrm{~s}$ faster than that of negative mass after the formation of negaitve and positive mass in nucleus field.

\section{ACKNOWLEDAGEMENT}

We would like to thank all the members if Innovative Ghar Nepal and Robotic Academy of Nepal, lalitipur-44700, Nepal to providing the different helps during our research works. Both financially and academically guidance during our research work.

\section{REFERENCES}

[1] L. Roszkowski, "Introduction to PHY008: Atomic and Nuclear Physics", p7, 2009/2010.

[2] M. Ferrario, M. Migliorati, and L. Palumbo, "Space Charge Effects", Proceedings of the CAS-CERN Accelerator School: Advanced Accelerator Physics, p331, 2014. Edited by W. Herr.

[3] K. Wiesemann, "A Short Introduction to Plasma Physics", AEPT, Ruhr-Universität Bochum, Germany p4-5.

[4] A. Lawncizak and V. Asbrink, "On the 1932 Discovery of the Positron", Royal Institute of Technology, SA104X Degree Project in Engineering Physics, First Level, Stockholm, Sweden, p10, 2014.

[5] W.S. Hwash, R. Yahaya, S. Radiman, "Effect of Core Deformation on 17B Halo Nucleus", Physics of Atomic Nuclei, Vol.77, Iss. 3, p275-281, 2014.

[6] A. Ozawa, T. Kobayashi, H. Sato and et al., "Interaction cross sections and radii of the mass number $A=17$ isobar $\left({ }^{17} \mathrm{~N},{ }^{17} \mathrm{~F}\right.$, and $\left.{ }^{17} \mathrm{Ne}\right)$ ", Physics Letters B, Vol.334, Iss.1-2, p18-22, 1994.

[7] M.A. Arrizabalaga, "Experimental Methods to Measure Nuclear Radii”, Física Nuclear Etsii, p4-17, 1993-94.

[8] E. Jiménez, N. Recalde and E.J. Chacón, "Extraction of the Proton and Electron Radii from Characteristic Atomic Lines and Entropy Principles", Entropy, Vol.19, Iss.293, p12, 2017.

[9] Physics 231, "Electric Charges, Forces, and Fields", p21, 2008.

[10] PHY 2049, "Chapter 22: Electrostatic Force and Electric Charge", p7.

[11] J.D. Cresser, "The Special Theory Of Relativity: Lecture Notes Prepared", Macquarie University, Sydeny, p.29, 2003.

[12] J. D. Cresser, "Lecture Notes on Special Relativity", Macquarie University Sydney, pp.38, August 18, 2005.

[13] P. Harris, "Lecture Note: Special Relativity", University of Sussex, pp.67-68.

[14] S.H. Dhobi, M.D.J. Rangrej, G.P. Adhikari, and M.N. Upadhyay,"Dipole and Mass Formation are two Phenomena Causing Formation of Particle and Antiparticle in Pair Production Phenomena", International Journal of Scientific Research in Physics and Applied Sciences, Vol.7, Iss.6, p16, 2019.

[15] D.C. Ghosh and R. Biswas, "Theoretical Calculation of Absolute Radii of Atoms and Ions. Part 1: The Atomic Radii", International Journal of Molecular Sciences, Vol. 3, p92, 2002.

[16] Eric Muller - Exploratorium, Scaling an Atom Nothing Matters, p1-2, 2002. 